\title{
Floating knee: epidemiological profile and management at the traumatology and orthopedics department (section A) of Hassan ii hospital, fez/ Morocco
}

\begin{abstract}
Introduction: The floating knee is an association an ipsilateral in the lower limb first described by BLAKE and MCBRYDE in 1974. Diagnosis is based on clinical and radiological findings. Management must be prompt, multidisciplinary and optimal. The aim of this study is to analyze the epidemiological, diagnostic and therapeutic aspect of this pathology.

Methods: we retrospectively reviewed the cases of 32 patients who were operated between January 2009 and November 2018. Only patients with a floating knee were included. Frazer's classification was used for staging and indexing. Severity of associated injuries was assessed using the Injury Severity Score as well as CauchoixDuparc classification to evaluate open fractures preoperatively. Karlstrom and Olerud criteria were used to evaluate functional results.

Results: Young active adults were the most affected with a mean age of 28 years and a clear male predominance $(90.62 \%)$. Road accidents were the main cause $(93.75 \%)$. According to Fraser's classification, $68.75 \%$ of cases were type I, type IIa (15.62 $\%)$, type IIb $(9.37 \%)$ and type IIc $(6.25 \%)$. Seventeen patients underwent IM nailing of both femur and tibia fractures. Four patients $(12.5 \%)$ underwent ORIF with DCP femur plate; 3 patients $(9.37 \%)$ underwent condylar plate bridging of the femur; whereas 2 patients $(6.25 \%)$ underwent ORIF of tibia with DCP plate and 2 patients $(6.25 \%)$ with T-buttress plate of the tibia plateau. Three patients benefitted from external fixation of tibia whereas, 1 patient underwent a femoral external fixation. The mean hospital -stay was 5 days and $68.75 \%$ of the patients had favourable outcome with bone consolidation.

Conclusion: The floating knee is an entity that is gradually becoming prevalent in our side of the globe usually involving young active adults. Diagnosis is radioclinical most often. Management is operative and should be carried by an experienced multidisciplinary team
\end{abstract}

Keywords: floating knee, polytrauma, fracture, reduction
Volume 10 Issue 4 - 2018

\author{
Kamal Lahrach,' Boubacar Soumaré,' Saeed \\ Abdul Razak,' Bah Souleymane,' Diarra \\ Abdoul Salam, ${ }^{2}$ Boutayeb Fawzi \\ 'Department of Trauma and Orthopedics of the Hassan II \\ Hospital, University Sidi Mohammed Ben Abdellah of Fez, \\ Morocco \\ ${ }^{2}$ Reference Health Center of Mopti, Mali
}

Correspondence: Abdoul Salam Diarra, Reference Health Center of Mopti, Mali, Tel +22365775204 , Email abdoulsalamdiarra@gmail.com

Received: August 14, 2018| Published: August 20, 2018

\section{Introduction}

The floating knee is an association of ipsilateral femur and tibia fractures first described by Blake \& Mcbryde. ${ }^{1}$ Often, the floating knee is a result of high-energy injury associated with multiple trauma and potentially life-threatening lesions. ${ }^{2}$ In more than $95 \%$ of cases, they are caused by Road traffic accidents (RTA's) with mortality believed to up 5-15\% in most European countries, due to the high incidence of associated lesions. ${ }^{3,4,5}$ Diagnosis is clinical with supporting imaging as part of initial workup. Management is difficult due to associated lesions thus requiring a multidisciplinary team. It must be prompt and optimal with key management goals centered around: resuscitation and stabilization of the vital functions of the severely injured, stable effective or definitive fixation, reduce hospital stay and prevent bedsores and accompanying thromboembolic disease through early weight bearing and physical therapy.

Since 2009, our expertise has seen significant improvement in this area due to an increase in number of cases managed at the Department Trauma and Orthopedics-A- of Hassan II Hospital in Fez.
Thus the authors intend to analyze the epidemiological profile, diagnostic and pathological challenges as well the management of what is a complex injury in young adults in the light of its functional outcome with reference to relevant literature.

\section{Patients and methods}

We retrospectively reviewed the files of patients managed by the Department of Trauma and Orthopedics A of the Hassan II University Hospital. From January 2009 to November 2018, 32 out of 60 trauma patients were referred to our department for severe knee trauma. All 32 patients were skeletally mature adults with floating knees. All patients were involved in high energy trauma with road traffic accidents being the cause. Patients were admitted to our unit through accident and emergencies department of the teaching hospital after initial resuscitation. A complete standard radiological assessment was routinely performed in all cases comprising a plain lower limb $\mathrm{x}$-rays spanning the hip and ankle joints with a pelvis AP view. Other imaging techniques such as cerebral CT, facial CT, angiography, full body $\mathrm{CT}$, ultrasound and other first line imaging tools were 
requested depending on clinical setting. Frazer's classification was used, like in most cases in literature, to classify fractures due to its simplicity, observerreliability and especially its prognostic value. Severity of associated injuries was assessed using the Injury Severity Score (ISS) ${ }^{6}$ whereas Cauchoix-Duparc classification helped assess soft tissue damage. Initial management entailed fluid resuscitation and stabilization of the severely injured as described in literature, ${ }^{7}$ although primary musculoskeletal care dependedon the severity of wounds and neurovascular damage:

i. For closed fractures with no neurovascular injury: lower limb splint or trans-calcaneal traction was prescribed to reduce pain and avoid complications.

ii. For open fractures: tetanus prophylaxis and intravenous antibiotics covering staphylococcus was administered prior to irrigation and debridement.

Pain relief is an essential component of early management. All patients received intravenous analgesics, thromboprophylaxis and methylprednisolone to prevent fat embolism. Close monitoring for compartment syndrome was done as part of preoperative planning.

Management is operative with single stage reduction of both fractures done in two operative times. Open fractures are the primary focus and should be fixed first.

For closed floating knees, operative chronology depended on the fracture and type fixation required. These include:

a. For closed reduction: intramedullary nailing, percutaneous screwing fixation (tibial plateau and femoral condyle fractures);

b. For open reduction internal fixation: tibia locking plates ( $\mathrm{T}$ buttress plate, dynamic compression (DCP) plates) and for the femur (DCP plate, condylar blade plates, distal locking plates (LCP), DCS plate screws)

All patients in our series commenced early postoperative rehabilitation depending on general conditions and physical state of patient as well as the stability of the implant used. Follow up required regular physical and routine $\mathrm{x}$-rays each month after surgery until bone union and every three months afterwards. To assess functional outcomes, like other authors, we used the Karlstrom and Olerud criteria (Figures 1-2). ${ }^{8}$


Figure I A-B, Preoperative $x$-rays (AP and lateral views) showing a Fraser type Ilb floating knee.


Figure $2 \mathrm{~A}-\mathrm{B}$, same fracture treated by ORIF by condylar locking plate and IM nailing of tibia respectively.

\section{Results}

The floating knee is a condition whose incidence has seen a considerable rise in our institution since 2009, going from only 3 cases in 2009 to 7 cases in 2016. A total of 60 patients with floating knees were admitted at the emergency department during this study period, 32 of whom were managed by the Department of trauma and Orthopedic Surgery A, Hassan II Teaching Hospital. Young active adults were the most affected with a mean age of 28 years and a clear male predominance $(90.62 \%)$. RTA's were the main cause of injury in $93.75 \%$ of cases with direct impact trauma reported in $81.25 \%$ of the patients. All patients were admitted through the accidents and emergencies department of the teaching hospital with an average admission time of 1.5 hours. On physical examination, $50 \%$ of our patients had an isolated floating knee, $25 \%$ had associated injuries whereas $25 \%$ were polytrauma patients. Open fractures were observed in 22 patients, $17(53.12 \%)$ in the leg and 5(15.62\%) in the femur. Leg wounds were type I in $60 \%$ of the cases, whereas type II and III wounds were $20 \%$ respectively. For femur fractures, $80 \%$ of the cases were type I wounds whereas $20 \%$ of the case were type II. With respect to wound complications, there were 3 cases $(9.37 \%)$ of vascular injury: one case was admitted with consumed ischemia of the lower extremity, one case of superficial femoral artery damage and one case with posterior tibial arterial injury. No nerve injuries were observed in our series. ISS score averaged 21 (head and neck (25\%), musculoskeletal(21.87\%), face $(9.37 \%)$, chest $(6.25 \%)$, and abdomino-pelvic(6.25\%) lesions. Routine musculoskeletal imaging with abdominal ultrasound was performed in $75 \%$ of our patients, cerebral CT scan in $25 \%$ of cases and a full body scans in $9.37 \%$ of cases. According to Fraser's classification $68.75 \%$ of cases were type I, $15.62 \%$ type IIa, $9.37 \%$ type IIb and $6.25 \%$ were type IIc floating knees. Nine patients were admitted to intensive care unit $(28.12 \%)$, including 08 critically injured polytrauma patients admitted for initial management and resuscitation. Only 1 patient required postoperative intensive care due to severe associated injuries. The mean stay in intensive care was 5.66days. Out of the subset of critically injured requiring resuscitation, we recorded 3 deaths. 5 patients underwent immediate fixation at the emergency operating room including one patient with a cerebral intra-parenchymal hematoma requiring evacuation and decompressive flap. The mean time to reduction was 
48hours, generally ranging from 2 hours to up to 144 hours. Patients were operated under spinal anesthesia in $68.75 \%$ of cases whereas general anesthesia was employed in $31.25 \%$ of cases. Seventeen patients underwent closed reduction by intramedullary nailing of both femur and tibia fractures, representing $53.12 \%$ of the cases. With respect to ORIF with locking plates, $4(12.5 \%)$ patients benefited from reduction using femur DCP plates, 3(9.37\%) patients with locking condylar plates whereas $2(6.25 \%)$ patients each underwent reduction of tibia fractures using DCP plate and T buttress plates respectively. For external fixation, $3(9.37 \%)$ patients underwent closed reduction of tibia fractures with external fixator whereas only $1(3.12 \%)$ patient underwent femur reduction involving external fixator. Mean hospital stay at the (trauma department was 05days.

Outcome was favorable and marked by bone union in $22(68.75 \%)$ patients. Time to union ranged from 3 to 4.6 months. Some of the complications included, among others, hemorrhagic shock in 5 patients leading to 3 deaths; a superficial femoral artery injury in 1 patient who underwent a repair; 1 case of leg ischemia due to a popliteal artery injury which required salvage trans-tibial amputation; a tibial artery lesion in 1 patient and 3 cases $(9.37 \%)$ of wound infection of a type II open femur fractures and two patients with infections of stage I open tibia fractures. Other complications like malunion (9.37\%), nonunion $(9.37 \%)$ and joint stiffness(3.12\%) were also noted. After a mean follow-up of 30months, functional results were excellent or good in $75 \%$ of our patients.

\section{Discussion}

The advent of several modes of transport coupled with the growth of the automobile industry and especially the non-compliance with the road safety regulations are largely to blame for the surge in road traffic accidents. In Morocco, RTA's are the main etiology of floating knees.

In our study, young male adults were the most affected with RTA the major cause. This is consistent with data from recent literature ${ }^{8-14}$ and could be due to the fact that young adult males are more active hence at a greater risk of RTA than other groups of society. Most series in literature reported wound complications in more than half of the cases ${ }^{8-14}$ which is similar to our study. In our study, these soft tissue injuries occur predominantly in the tibia compared to the femur, which can be explained by the anatomical situation of the 2 bones. Associated lesions are quite common with incidence of vascular injury ranging from $6.25 \%$ according to Fraser $^{9}$ up to $9.37 \%$ in our series. These lesions can compromise short term results with wound complications leading to amputation. No nerve injury was found in our series, unlike some cases reported in literature ${ }^{9,13-16}$ In our case, these lesions were ruled out on physical examination. Several associated injuries are reported by some authors given the impact of injury, setting of polytrauma and/or multiple fractures requiring multidisciplinary management ${ }^{8,10,12,14,17}$ In most of the series in literature, the proportion of extra-articular fractures in both bones is greater than articular knee fractures. There is also a greater incidence of shaft fractures compared to metaphyseal fractures in both bones (leg and thigh). Bifocal fractures of the tibia are very frequent according to some authors ${ }^{8,9,10,13,14,16,17}$ as was the case in our study where $68.75 \%$ of the cases involved were Fraser type 1 shaft fractures. All authors are unanimous on the need for urgent stable reduction. The mean time to reduction varies between 2 days for Rethman $U^{18}$ to 5.2 days for BEHRMAN SW and coll. ${ }^{19}$ In our case series it was 2days. With regards to reduction, IM nailing was the most common method of fixation with $75 \%$ of femur fractures and $68.75 \%$ for tibia fixation, as reported by many other authors. ${ }^{10,12,13,17}$ However, in some studies, locking plates were preferred. ${ }^{11,16}$

Associated injuries are a common cause of death in polytrauma patients. We recorded $9.37 \%$ in our series compared to $4.05 \%$ and $1.75 \%$ per Fraser RD and Veith Rg respectively., ${ }^{9} 10$ Infection rates reported by Fraser $\mathrm{Rd}^{9}$ were significantly higher than those observed in our study ( $30 \%$ versus $9.37 \%$ ). This could be explained by the routine use of intravenous antibiotic prophylaxis as part of initial management of severe knee fractures. Salvage amputation according to some authors, ${ }^{10,17}$ could be necessary due to either failure of revascularization after vascular repair or severe wound infection with gangrene. Its frequency was $3.12 \%$ in our series compared to $1.7 \%$ and $5.5 \%$ according to Veith $\mathrm{RG}^{10}$ and Hung Tk. ${ }^{17}$

Early administration of methylprednisolone is believed to reduce the risk off at embolism and no such complication was observed in our study. A few cases exist in literature with a relatively lower rate of fat embolism. ${ }^{8-14}$ Deep Venous thrombosis and postoperative pulmonary embolism are becoming rare due to the availability of effective means of prevention as well as improved methods that allow early weight bearing. HUNG SH an-d ZRIG M, reported malnutrition rate of 5.5\% and $46.1 \%$ respectively, whereas it was $9.37 \%$ in our series. Knee stiffness remains a major drawback of floating knees thus should be targeted by early well prescribed rehabilitation. Few cases of knee stiffness $(3.12 \%)$ was observed in our study could be related to early physical therapy and range of motions encouraged in our department. FRASER.R.D reported nonunion rate of $14.9 \%{ }^{9}$ and VEITH.RG observed a nonunion rate of $7.3 \%{ }^{10}$ whiles in our series $9.37 \%$ of cases presented with nonunion. Overall functional outcomes could be improved by stable reduction allowing early adapted range of motion and rehabilitation; as evidenced by good and excellent functional results according to Karsltrom criteria. Thus our study goes to add to the current level of evidence in literature ${ }^{13,16,20}$ that suggests that the outcome of floating knees is largely dependent on the Frazer's classification and the type of wound as major prognostic factors.

\section{Conclusion}

The floating knee is an entity whose incidence is gradually becoming frequent in our side of the globe that could threaten the lives of young male adults. Its diagnosis is often radio-clinical most often. Management is surgical often requiring an experienced multidisciplinary team. Operative treatment should be early to reduce the risks of complication and guarantee good functional outcome.

\section{Acknowledgements}

None.

\section{Conflict of interest}

Author declares that there is no conflict of interest.

\section{References}

1. Mc Bryde Am, Blake R. The floating knee: ipsilateral fractures of the femur and tibia. J Bone and Joint 1974;56-A, Nº, September: 1309

2. Titcheu F, Bintou S, Bayiha D. Le genou flottant : formes cliniques, traitements et résultats à propos de 30 cas. Orth Emerg Afr. 2012.

3. Fraser RD, Hunter GA, Waddell JP. Ipsilateral fracture of the femur and 
tibia. J Bone Joint Surg. 1978;60:510-515.

4. Piétu G, Jacquot F, Féron J-M. et les membres du GETRAUM The floating knee: a retrospective analysis of 172 cases. Revue de chirurgie orthopédique. 2007.

5. Le genou flottant: A propos de 30 cas Thèse de médecine $N^{\circ} 26 / 2011$ Université CADI AYYAD - faculté de médecine et de pharmacie.

6. Baker SP, O'neill B, Haddon W. The injury severity score: a method for describing patients with multiple injuries and evaluating emergency care. J Trauma. 1974;14:187-196.

7. Feron Jm, Signoret F, Bonfait H. Patel A Le genou flottant: indication thérapeutique et résultats Communication orale 8ème congrès international de chirurgie orthopédique de la Méditéranée, Montpelier. 1985:16-18.

8. Karlstrom G, Olerud S. Ipsilateral fracture of the femur and tibia. J Bones Joint Surg. 1977:59;240-243.

9. Fraser Rd, Hunter GA, Wadell JP. Ipsilateral fracture of the femur and tibia. J Bone and Joint. 1978;60-B, N4:510-515.

10. Veith RG, Winquist RA, Hansen ST. Ipsilateral fractures of the femur and tibia. J Bone and Joint. 1984;66-A, No7:991-1002.

11. Hwan Tak H, Ho Poh W, Yin Peng L. Leann M Predictors of outcome of floating knee injuries in adults. Acta Orthop Scand. 2001;72:385-394.

12. Rethman U, Yesupalan RS, Nair. The floating knee: epidemiology, prognosis indicators and outcome following surgical management. $J$ Trauma Manag Outcomes. 2007;1(1):2.
13. Pietu G, Jacquot F, Freron JM. The floating knee: a retrospective analysis of 172 cases. Rev Chir Orthop Reparatrice. 2007;93(6):627-634.

14. Zrig M, Mnif H, Hammouda I. Le genou flottant: Etude rétrospective de 39 cas Tunisie Orthopédie. 2008;1, N²:165-170.

15. Ya Liu, Jun Zhang, Shu Zhang. Concomitant ligamentous and meniscal injuries in floatting knee Department of orthopedics and traumatic surgey, Jinan Military General Hospital, China; 2015.

16. Marco FA, Rozim AZ, Piedade Sr. Knee joint stability in a "floating knee" condition. Acta Ortop Bras. 2008;16(1):32-36.

17. Hung SH, Lu YM, Hvang HT, et al. Surgical treatment of type II of floating knee: comparaison of the results of type IIa and type IIb floating knee Knee surg sports traumatol arthrosc. 2007;15(5):578-586.

18. Bone Lb, Johnson Kd, Weigelt J. Early versus delayed stabilization of femoral fractures: a retrospective randomized study. $J$ Bone and Joint. 1989;71-A, $\mathrm{N}^{\circ} 3: 336-340$.

19. Behrman SW, Fabian TC, Kudsk KA. Improved outcome with femur fractures: early versus delayed fixation. J Trauma. 1990;30:792-798.

20. Niya F. Genou flottant à propos de 24 cas. Thèse médicale. $\mathrm{N}^{\circ} 308,2004$ 